\title{
Effect of Heat Transfer on the Efficiency of Micro Size Turbine and Compressor Used in Turbocharger
}

\author{
Omer Faruk Atac', Jeong-Eui Yun ${ }^{1}$, Taehyun Noh ${ }^{2}$ \\ ${ }^{1}$ Dept. of Mechanical Design Engineering, Kangwon National University \\ 346 Joongang-ro, Samcheok-si, 25913 Republic of Korea \\ o.farukatac@gmail.com, jeyun@kangwon.ac.kr \\ ${ }^{2} \mathrm{R} \& D$ Center, Keyyang Precision Co. Ltd. \\ 32 Gongdan 4 gil, Gimcheon-si, 39537, Republic of Korea \\ th_noh@keyyang.co.kr
}

\section{Extended Abstract}

The main aim of this study is to correct the efficiency of turbine and compressor due to the heat transfer phenomenon on the turbocharger, in particular, between the impellers (turbine and compressor) and other surroundings (volutes). Until today, several mathematical models were proposed to detect the diabatic conditions on the turbocharger based on the gas stand, lumped capacitance, or numerical methods[1-3]. In this study, the influence of heat transfer on the radial compressor and turbine performance was investigated by steady-state conjugate heat transfer (CHT) simulations and compared with adiabatic conditions. In order to correct the impeller efficiencies, shaft work on the turbine was measured and used as diabatic work done on the corrected efficiency equations. This is because heat transfers (before/after the compression/expansion) already took into account by CHT simulations. Therefore, shaft power is given by the remaining exhaust gas energy (after heat transfer) on the turbine. The results indicated that compressor efficiency was significantly affected due to heat flux between diffuser and center case. It also means that heat transfer mainly occurred after the compression process. Based on the correction, approximately $11 \%$ efficiency drop was observed on the compressor in comparison to the adiabatic condition. Regarding the turbine, heat transfer mostly takes place between the exhaust gas and volute-surroundings before the expansion process and $12 \%$ efficiency reduction was detected. As a result, we could say that heat transfer on the micro size turbine and compressor used in turbocharger plays an important role for turbocharger-engine matching due to significantly differences between corrected performance maps and manufacturer maps.

\section{Acknowledgement}

This research was supported by the Center for Environmentally Friendly Vehicles (CEFV) as the Global-Top Project of the Ministry of Environment, Korea (KMOE).

\section{References}

[1] A. Romagnoli, A Manivannan, S. Rajoo, M. S. Chiong, A. Feneley, A. Pesiridis and R. F. Martinez-Botas, "A review of heat transfer in turbochargers," Renew. Sustain. Energy Rev., vol. 79, no. April, pp. 1442-1460, 2017.

[2] L. Gu, A. Zemp, and R. S. Abhari, "Numerical study of the heat transfer effect on a centrifugal compressor performance," Proc. Inst. Mech. Eng. Part C J. Mech. Eng. Sci., vol. 229, no. 12, pp. 2207-2220, 2015.

[3] R. D. Burke, C. R. M. Vagg, D. Chalet, and P. Chesse, "Heat transfer in turbocharger turbines under steady, pulsating and transient conditions," Int. J. Heat Fluid Flow, vol. 52, pp. 185-197, 2015. 